\title{
New Compounds against Leishmania infantum from Eremurus persicus ${ }^{\dagger}$
}

\author{
Emanuela Martino ${ }^{1, *}$, Valeria Cavalloro ${ }^{1,2}$, Elisa Vignoni ${ }^{2}$, Serena Della Volpe ${ }^{2}$, Karzan M. Ahmed ${ }^{3}$, \\ Maria Do Céu Sousa ${ }^{4}$, Daniela Rossi ${ }^{2}$ and Simona Collina ${ }^{2}$ \\ 1 Earth and Environmental Sciences Department, University of Pavia, Via S. Epifanio 14, 27100 Pavia, Italy; \\ valeria.cavalloro01@universitadipavia.it \\ 2 Department of Drug Sciences, University of Pavia, Via Taramelli, 12, 27100 Pavia, Italy; \\ elisa.vignoni01@universitadipavia.it (E.V.); serena.dellavolpe@gmail.com (S.D.V.); \\ daniela.rossi@unipv.it (D.R.); simona.collina@unipv.it (S.C.) \\ 3 Faculty of Education, School of Science, Chemistry Department, University of Garmian, 46021 Kalar, \\ Kurdistan; karzchem@yahoo.com \\ 4 Faculty of Pharmacy, University of Coimbra, Pólo das Ciências da Saúde, Azinhaga de Santa Comba, \\ 3000-548 Coimbra, Portugal; mcsousa@ci.uc.pt \\ * Correspondence: emanuela.martino@unipv.it \\ + Presented at the 2nd Molecules Medicinal Chemistry Symposium (MMCS): Facing Novel Challenges in \\ Drug Discovery, Barcelona, Spain, 15-17 May 2019.
}

Published: 15 August 2019

Keywords: Leishmaniasis; Eremurus persicus; (R)-ASME; Nature Aided Drug Discovery

Leishmaniasis is a complex of vector-borne diseases caused by several species of the protozoan parasite of the genus Leishmania. This disease affects 12 million people in the world, and its burden is particularly felt in undeveloped and developing countries. To date, a vaccine has not been discovered yet; thus, chemotherapy remains the only effective way to treat every form of the disease. However, many available drugs have the drawback of causing both serious side effects and toxicity, and resistance. Accordingly, there is an urgent need of new effective drugs.

Within a wider research project aimed at discovering new bioactive compounds from nature, for several years we have focused our attention on plants from the Asian region and particularly on Eremurus persicus. Following a bio-guided fractionation approach, we isolated (R)-Aloesaponol III 8methyl ether $(R)$-ASME, characterized by a remarkable antiprotozoal effect against L. infantum $(\mathrm{IC} 50=73 \mu \mathrm{g} / \mathrm{mL})$. However, $(R)$-ASME has a very low solubility both in water and in aqueous buffers, and therefore, the biological assays can be difficult to perform and time-consuming, since a high number of replicates are needed. Starting from $(R)$-ASME as a hit compound, we designed new water-soluble $(R)$-ASME- derivatives. Taking into account its structural features and molecular reactivity, molecular modification strategies have been planned, including conjugation of $(R)$-ASME with either amino acid (AA), or with PEG moiety. The latter approach allowed us to obtain a stable and water soluble salt. The assays against Leishmania infantum are still in progress.

(C) 2019 by the authors. Licensee MDPI, Basel, Switzerland. This article is an open access article distributed under the terms and conditions of the Creative Commons Attribution (CC BY) license (http://creativecommons.org/licenses/by/4.0/). 